\title{
Precision medicine in early-onset epilepsy: the KCNQ2 paradigm
}

\author{
Elisabetta Amadori ${ }^{1,2, \star}$, Noemi Brolatti ${ }^{2, \star}$, Marcello Scala ${ }^{1}$, Francesca Marchese ${ }^{2}$, Maria Stella Vari ${ }^{2}$, Luca \\ Antonio Ramenghi ${ }^{3}$, Francesca Madia ${ }^{4}$, Carlo Minetti ${ }^{1,2}$, Pasquale Striano ${ }^{1,2}$ \\ 'Department of Neurosciences, Rehabilitation, Ophthalmology, Genetics, Maternal and Child Health, University of Genoa, \\ Genoa 16147, Italy. \\ 2Pediatric Neurology and Muscular Diseases Unit, IRCCS ‘G. Gaslini' Institute, Genoa 16147, Italy. \\ ${ }^{3}$ Neonatal Intensive Care Unit, IRCCS 'G. Gaslini' Institute, Genoa 16147, Italy. \\ ${ }^{4}$ Unit of Medical Genetics, IRCCS 'G.Gaslini' Institute, Genoa 16147, Italy. \\ ${ }^{\star}$ Equally contributing authors.
}

Correspondence to: Prof. Pasquale Striano, Department of Neurosciences, Rehabilitation, Ophthalmology, Genetics, Maternal and Child Health, University of Genoa, Genoa 16147, Italy; Pediatric Neurology and Muscular Diseases Unit, IRCCS 'G. Gaslini' Institute, Via Gerolamo Gaslini 5, Genoa 16147, Italy. E-mail: strianop@gmail.com

How to cite this article: Amadori E, Brolatti N, Scala M, Marchese F, Vari MS, Ramenghi LA, Madia F, Minetti C, Striano P. Precision medicine in early-onset epilepsy: the KCNQ2 paradigm. J Trans/ Genet Genom 2020;4:278-84.

http://dx.doi.org/10.20517/jtgg.2020.36

Received: 31 May 2020 First Decision: 30 Jun 2020 Revised: 6 Jul 2020 Accepted: 10 Jul 2020 Available Online: 26 Jul 2020

Academic Editor: Ramón Cacabelos Copy Editor: Cai-Hong Wang Production Editor: Tian Zhang

\begin{abstract}
The identification of the genetic causes and the underlying pathogenic mechanisms in early-onset epilepsies has proved to be essential in improving the efficacy of therapeutic decisions and the overall patient management, especially in the era of precision medicine. We report an infant presenting with a cluster of focal motor seizures with autonomic manifestations at day 3 of life. Electroencephalograms showed multifocal epileptic abnormalities and a burst-suppression pattern. Neurological examination showed poor visual fixation and hypotonia. Neuroimaging was normal. Seizures remitted with phenytoin and were well-controlled after the switch to oral carbamazepine. In the hypothesis of a genetic etiology, next-generation sequencing panel for epileptic encephalopathies was performed and identified a de novo missense mutation in KCNQ2: c.1742G>A; p.(Arg581GIn) (NM_172107.2). This case report highlights the importance of the early recognition of the electroclinical phenotype and the detection of the underlying genetic cause in the implementation of "tailored" therapies in early-onset genetic epilepsies.
\end{abstract}

Keywords: KCNQ2, early-onset epilepsy, developmental and epileptic encephalopathy, next-generation sequencing, precision medicine, sodium channel blockers 


\section{INTRODUCTION}

Epilepsy is one of the most common neurological disorders. It is more frequent in infancy with an incidence of approximately 70 per 100,000 children younger than 2 years ${ }^{[1]}$. Approximately $70 \%-80 \%$ of childhood epilepsy cases are caused by one or more genetic alterations, which comprise both monogenic and polygenic factors $^{[2]}$. While the majority of seizures occurring during the neonatal period are caused by acute brain injury ${ }^{[3]}$, around $13 \%$ of neonates suffer from neonatal-onset epilepsy ${ }^{[4]}$ with a specific genetic cause, usually showing up in the first days of life.

Neonatal-onset genetic epilepsies can be classified into three large groups: genetic conditions associated with structural brain anomaly, genetic conditions associated with metabolic disorders, and genetic conditions resulting in functional cortical anomalies ${ }^{[3]}$. The latter group includes disorders of cell signalling (e.g., CDKL5, BRAT1), disorders of synaptic transmission (e.g., STXBP1), and channelopathies (e.g., KCNQ2, $K C N T 1, S C N 2 A)^{[3]}$. Pathogenic variants in $K C N Q 2$ are the most common genetic cause of neonatal seizures among the channelopathies ${ }^{[5]}$. Dominant mutations in this gene lead to different epileptic disorders, including benign epilepsy and developmental and epileptic encephalopathy $(K C N Q 2-\text { related DEE })^{[6]}$. The clinical features suggestive of KCNQ2-related DEE include seizure onset in the first week of life with severely abnormal electroencephalography background activity, absence of structural lesions in the brain, and mild to severe developmental delay ${ }^{[6,7]}$.

Our understanding of the etiology and pathophysiology of DEE has considerably improved thanks to Next Generation Sequencing (NGS). This technique has become faster and more accessible in recent years ${ }^{[8]}$, allowing the development of treatments addressing the precise molecular causes of the disease ${ }^{[8]}$. Early genetic diagnosis is critical to avoid further potentially invasive diagnostic tests and to make appropriate therapeutic decisions. The case of KCNQ2 related-epilepsy is paradigmatic, as supported by the importance of the early recognition of the specific electroclinical phenotype and the use of sodium channel blockers (SCB) in this disorder ${ }^{[9]}$.

We describe a boy with $K C N Q 2$-related DEE presenting with neonatal-onset seizures; the epilepsy genepanel revealed a de novo KCNQ2 variant: c.1742G>A; p.(Arg581Gln) (NM_172107.2). We highlight the immediate and remarkable response to the treatment with SCB in this patient, further supporting the role of this therapeutic approach in KCNQ2 disorders.

\section{CASE REPORT}

This 7-month-old infant was the first child from unrelated, healthy parents. Family history was negative for epilepsies, mental retardation, and hereditary diseases, but it was positive for febrile seizures on the maternal side. He was born at term by urgent caesarean section for deceleration of the heartbeat, after an uneventful pregnancy. Fetal karyotype was normal. APGAR score was 8 at $1 \mathrm{~min}$ and 9 at $5 \mathrm{~min}$. Auxological parameters at birth were: weight 2,650 g (-2.2 Standard Deviation - SD, SGA), length $52 \mathrm{~cm}(+1.13 \mathrm{SD})$, and occipital frontal circumference (OFC) $36 \mathrm{~cm}(+1.41 \mathrm{SD})$. Transient neonatal hypoglycemia was found, but the perinatal course was otherwise uneventful.

At day 3, the baby presented recurrent seizures, lasting around one minute and characterized by tonic posturing with flexion of the lower limbs, ocular retroversion, horizontal nystagmus, perioral cyanosis, automatic sucking movements, and apnea (with $\mathrm{SaO}_{2}<45$ ). He was hospitalized in the Neonatal Intensive Care Unit and a serial electroencephalographic (EEG) monitoring was performed. The first EEG showed rare paroxysmal abnormalities in the left hemisphere. Due to the daily recurrence of clustered focal motor and autonomic seizures, the patient was treated with intravenous phenobarbital (PB) at the initial dose of $20 \mathrm{mg} / \mathrm{kg}$ up to the maintenance dose of $5 \mathrm{mg} / \mathrm{kg}$ twice daily, with no effect. At day 6, levetiracetam 
A

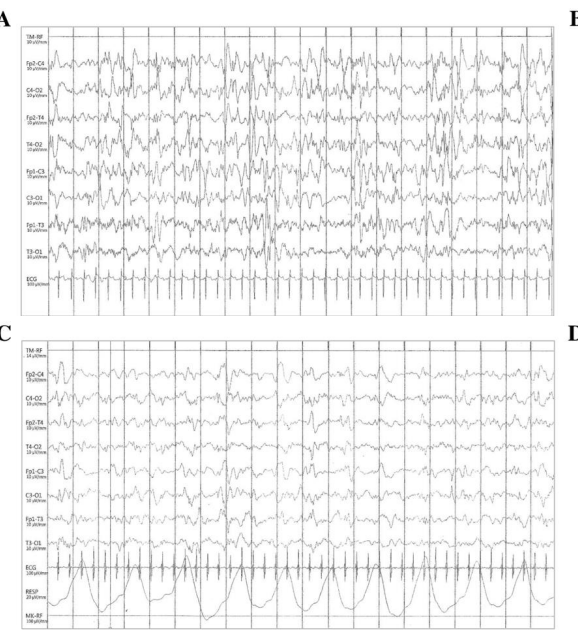

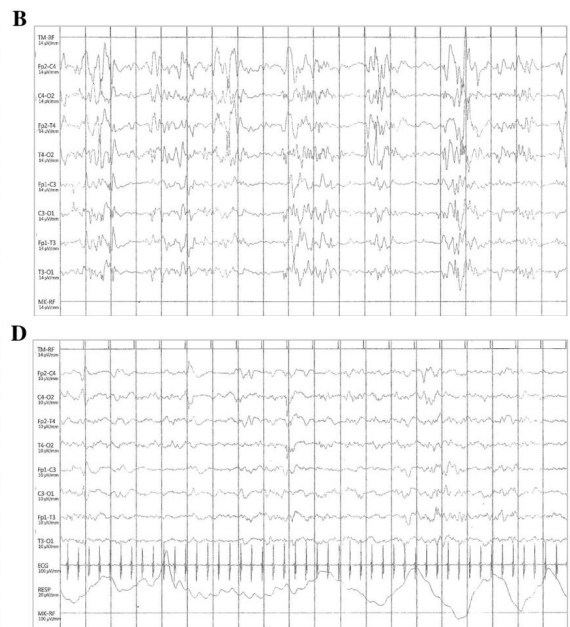

Figure 1. $E E G$ findings in the patient. $A$ : EEG at 13 days showing multifocal epileptic abnormalities; $B$ : EEG at 18 days shows suppression burst activity; C: EEG during sleep at 25 days after the introduction of sodium channel blockers reveals an attenuation of multifocal epileptiform abnormalities; D: EEG in wake with sporadic epileptic abnormalities and monomorphic theta-delta rhythms, combined with slow bifrontal graphoelements. All EEG shown are in a reduced montage with setting the low-frequency time constant at 0.1 seconds, high-frequency filter at $50 \mathrm{~Hz}$ (except in frame B where it is $15 \mathrm{~Hz}$ ), notch filter at $50 \mathrm{~Hz}$, sensitivity at $10 \mu \mathrm{V} / \mathrm{mm}$ (except in frame B: $14 \mu \mathrm{V} / \mathrm{mm})$, paper speed is $20 \mathrm{sec}$ per epoch

(LEV) $(16 \mathrm{mg} / \mathrm{kg} /$ twice per day) was added-on with partial response. Neurological examination revealed poor visual fixation, generalized hypotonia, right head deviation, and opisthotonus. Biochemical workup (serum electrolytes and ammonium, glucose level, coagulation profile, plasma, and urinary amino acids, acylcarnitine, and urinary organic acids) and extensive screening for possible infections in blood and cerebrospinal fluid were unremarkable. Electrocardiogram, echocardiogram, abdomen ultrasonography, and auditory brainstem evocative potentials were normal. Brain magnetic resonance imaging, including spectroscopy and angio sequences, was unremarkable at age 13 days.

Seizures recurred with axial hypertonia, extension of the four limbs, deviation of the head to the right side, perioral cyanosis, and occasional desaturation. A second EEG at 13 days of age showed relevant multifocal epileptic abnormalities and no significant change was observed after a trial with $100 \mathrm{mg}$ pyridoxine bolus [Figure $1 \mathrm{~A}$ ]. Furthermore, seizures persisted despite the administration of intravenous midazolam at maximum dosages and pyridoxine, in add-on with PB and LEV. Likewise, the introduction of clobazam (5 mg/die) was ineffective. At 18 days, the EEG recording confirmed a significant multifocal paroxysmal activity. A burst suppression activity was also observed [Figure 1B]; moreover, amplitude-integrated EEG (aEEG) monitoring showed a pattern with "peaks" and subsequent depression.

Thus, genetically determined epilepsy with neonatal-onset was suspected. After written informed consents obtained from the parents, DNA was extracted from the peripheral blood of the trio and NGS-based panel including 54 genes involved in epileptic encephalopathies was performed at twenty days of life (full list of genes is available on Supplementary Table 1).

Intravenous phenytoin (PHT) was started and then switched to oral, gradually adjusting the dosages based on the blood levels. The final dosage was $40 \mathrm{mg} / \mathrm{kg} / \mathrm{day}$ divided into three administrations. This therapy did control the seizures and the other antiepileptic medications $\left(\mathrm{VB}_{6}, \mathrm{LEV}, \mathrm{PB}\right.$ and benzodiazepines) were gradually withdrawn. The EEG recording at 25 days of age still showed multifocal epileptiform activity in sleep, with alternating trends [Figure $1 \mathrm{C}$ ]. These abnormalities were attenuated during wake and were associated with medium voltage monomorphic theta-delta rhythms with slow bifrontal graphoelements. At age 1 month, the EEG revealed better-organized background activity and a reduction in the epileptic 
activity [Figure 1D]. At 36 days of age, PHT blood levels were found to be inappropriately low $(0.9 \mu \mathrm{g} / \mathrm{mL}$, reference range: $10-20 \mu \mathrm{g} / \mathrm{mL}$ ) and electroclinical worsening was observed. Accordingly, the PHT dosage was increased up to $45 \mathrm{mg} / \mathrm{kg} /$ day, in three daily administrations. Background EEG activity was replaced by abnormal activity which included spikes and polyspikes, often followed by slow waves of medium amplitude, which were synchronous and asynchronous in both hemispheres and more prominent in the frontal derivations. Sometimes these abnormalities were more diffuse. Additional EEGs performed at 2 months of life showed a significant improvement. There were rare epileptic abnormalities with multifocal expression and predominant posterior origin.

Due to the confirmation of a heterozygous missense mutation in KCNQ2 [c.1742G>A; p.(Arg581Gln)] absent in the parents (de novo origin), we introduced carbamazepine (CBZ) at the initial dosage of $9 \mathrm{mg} / \mathrm{Kg} / \mathrm{day}$, progressively increasing up to an adequate dosage for body weight. This change in the therapeutic regimen resulted in good seizures control. Two further EEGs (at the age of 70 and 85 days), showed a lack of organization and multifocal epileptic abnormalities. The baby was discharged from hospital at the age of 3 months. He was seizure-free with CBZ monotherapy.

At 7 months of age, the patient experienced daily episodes of apnea and cyanosis preceded by crying. Video-EEG showed multifocal epileptic abnormalities and confirmed the epileptic nature of these episodes. Furthermore, the CBZ blood levels were $2.4 \mu \mathrm{g} / \mathrm{mL}$ (reference range: $8-12 \mu \mathrm{g} / \mathrm{mL}$ ). The dosage was thus increased $(16.7 \mathrm{mg} / \mathrm{kg} / \mathrm{die})$ with subsequent good seizures control. Physical examination revealed frontal bossing, irritability, mild hypertonia, vocalizations, and inconstant visual fixation. Psychomotor delay was observed, as he was not able to sit unassisted. Auxological parameters were: weight of $7.2 \mathrm{~kg}(-1.64$ $\mathrm{SD})$, length of $64 \mathrm{~cm}(-2.05 \mathrm{SD})$, OFC $46 \mathrm{~cm}(+1.75 \mathrm{SD})$. Head ultrasound showed a $6 \mathrm{~mm}$ dilation of the periencephalic spaces of the cranial vault.

\section{DISCUSSION}

KCNQ2 is located on chromosome 20q13.33 and encodes the potassium channel subunit $\mathrm{K}_{\mathrm{v}}$ 7.2., that forms heterotetrameric voltage-gated potassium channels together with $\mathrm{K}_{\mathrm{v}} 7.3$ subunit (encoded by KCNQ3). These channels are widely expressed in the brain and transport potassium ions along their concentration gradient, underlying the $\mathrm{M}$-current. This slowly activating, non-inactivating potassium conductance inhibits neuronal excitability ${ }^{[10]}$. Pathogenic variants in KCNQ2 leading to loss of function cause two different epilepsy phenotypes ${ }^{[6]}$ Truncating and missense variants, likely resulting in haploinsufficiency, are associated with benign familial neonatal epilepsy (BFNE) whereas missense variants and small in-frame deletions, resulting in dominant-negative effect, are associated with $\mathrm{DEE}^{[6,11]}$. Hereditary or de novo BFNE-associated KCNQ2 variants are distributed along with the channel protein and are more common in the intracellular domain between $\mathrm{S} 2$ and $\mathrm{S}^{[12]}{ }^{[1 .}$. The mutations in $\mathrm{KCNQ} 2$ in encephalopathic patients are instead found in four functionally relevant protein domains: the domain of the voltage sensor, the pore, the proximal regions C-terminus, and calmodulin-binding helix region $\mathrm{B}^{[7,12]}$. The variant detected in our case is reported in the ClinVar database in an individual with neonatal/infantile-onset epileptic encephalopathy (\#ClinVar, variation ID 21769, https://www.ncbi.nlm.nih.gov/clinvar/variation/21769/). This variant involves an evolutionarily conserved amino acid located in the C-terminus domain, likely resulting in an impaired protein function.

The recognition of seizures in a new-born can be challenging as clinical manifestations may be subtle. The EEG background plays a relevant role in the identification of patients with benign epilepsy or severe epilepsy. Indeed, video-EEG is a powerful tool for the early recognition of distinct electro-clinical aetiology-specific phenotypes. This is further supported by what has been observed in KCNQ2-related epilepsies, KCNT1 encephalopathy, and $S T X B P 1$ encephalopathy ${ }^{[13]}$. In our patient, the neonatal EEG background showed multifocal epileptic abnormalities with random attenuation alternating with burst suppression pattern. Moreover, aEEG showed a pattern that has been recently suggested as a signature in neonates with KCNQ2related disorders ${ }^{[14]}$. 
Deep phenotypic characterization played a relevant role in the choice of the most appropriate genetic test in our case. Genetic testing should be performed as early as possible, especially in patients showing some specific electro-clinical phenotypes (e.g., KCNQ2 or KCNT1), to recognize potentially treatable disorders ${ }^{[15]}$.

In our patient, genetic testing was performed within the first month of life and the results were obtained at age 2 months, revealing the de novo missense mutation in KCNQ2 and allowing a "targeted" treatment.

The role of a precision medicine approach in neonatal seizures with known etiology is becoming more and more relevant in recent years ${ }^{[13,16]}$. In consideration of the good electro-clinical response to SCB, our suspicion was focused on $S C N 2 A, S C N 8 A$, and KCNQ2.

Voltage-gated sodium channels and KCNQ potassium channels co-localize at the neuronal membrane and a modulating effect of SCB on both channels has been suggested ${ }^{[17]}$. The use of broad-spectrum antiepileptic drugs in KCNQ2-related epilepsy has been suggested ${ }^{[9]}$. The most common drug used in "benign" phenotypes was $\mathrm{PB}$, followed by $\mathrm{SCB}^{[9]}$. In general, patients with a mutation in $K C N Q 2$ suffering from $\mathrm{B}(\mathrm{F})$ $\mathrm{NE}$ become seizure-free spontaneously in the first month of life or between the 6 months and 1 year of age. Accordingly, the International League Against Epilepsy recommends the use of the term "self-limiting" instead of "benign" ${ }^{n[18]}$. Therefore, the large use of PB is most likely explained by the common use of this drug as standard therapy in neonatal seizures ${ }^{[9]}$. CBZ and PHT are instead considered appropriate as firstline treatment in KCNQ2-related encephalopathy, regardless of the underlying pathogenic variant ${ }^{[\rho]}$. Also, lacosamide improved seizures frequency in the early-onset epileptic encephalopathy ${ }^{[9]}$. However, functional studies and additional clinical observations are required before it can be considered a possible target therapy $^{[19]}$.

The finding that retigabine opens the $\mathrm{K}_{\mathrm{v}} 7$ potassium channel sparked the interest in its use in patients with a KCNQ2 mutation and it was considered the first specific anti-seizure drug for KCNQ-related epilepsy. Despite preliminary promising results, especially when the drug was administered during early development (such as improvement of seizure frequency and development in patients treated before the age of 6 months ${ }^{[12]}$, its prescription was questioned because long-term treatment was characterized by several side effects (e.g., blue discolouration of skin and retina) ${ }^{[20]}$.

Another possible drug in patients with abnormal KCNQ function is pyridoxine. Indeed, some patients with KCNQ2-encephalopathy respond to this vitamin but the pathophysiology of mechanisms behind this response to pyridoxine is not completely understood ${ }^{[21]}$.

In our patient, we switched to CBZ after the dramatic effect of PHT treatment on seizure control because of the safer drug profile of CBZ within the SCB group. Indeed, other SCBs present several disadvantages, as shown by the adverse event profile of PHT (purple glove syndrome, cerebellar toxicity, and cardiac arrhythmias) or long-lasting dose increments of lamotrigine ${ }^{[9]}$.

Current treatments in KCNQ2-related epilepsy mainly focus on seizure control, but the early genetic diagnosis will be more and more relevant in the choice of the most appropriate therapeutic regimen, especially considering the progressive introduction of new therapies.

Advances in the knowledge of the genetic basis of early-onset epilepsies have opened the way for precision medicine approaches in this category, which had been long considered as a single entity. In KCNQ2related encephalopathy, seizures may remit, but mild to profound global developmental delay may occur ${ }^{[18]}$. Therefore, it is mandatory that in the future, clinicians will consider to run genetic testing as part of the initial workup, with higher priority for neonates, so that the results can be incorporated in the early 
treatment decisions. This would, for instance, allow selecting SCBs from the beginning and avoid the usual trial-and-error approach that was also applied in our patient.

\section{DECLARATIONS}

\section{Acknowledgments}

This work was developed within the framework of the DINOGMI Department of Excellence of MIUR 20182022 (legge 232 del 2016).

\section{Authors' contributions}

Drafted the manuscript: Amadori E, Brolatti N

Collected data: Brolatti N, Marchese F, Vari MS

Analyzed and interpreted the electroclinical data: Amadori E, Marchese F, Vari MS

Analyzed genetic data: Madia F

Critically reviewed the manuscript: Scala M, Ramenghi LA, Minetti C, Striano P

\section{Availability of data and materials}

The datasets generated for this study can be found in Pediatric Neurology and Muscular Diseases Unit and Neonatal Intensive Care Unit of 'G. Gaslini' Institute.

\section{Financial support and sponsorship}

None.

\section{Conflicts of interest}

All authors declared that there are no conflicts of interest.

\section{Ethical approval and consent to participation}

Not applicable.

\section{Consent for publication}

Not applicable.

\section{Copyright}

(c) The Author(s) 2020.

\section{REFERENCES}

1. Orsini A, Zara F, Striano P. Recent advances in epilepsy genetics. Neurosci Lett 2018;667:4-9.

2. Hildebrand MS, Dahl HH, Damiano JA, Smith RJ, Scheffer IE, et al. Recent advances in the molecular genetics of epilepsy. J Med Genet 2013;50:271-9.

3. Cornet M, Cilio MR. Genetics of neonatal-onset epilepsies. Neonatal Neurology. Elsevier; 2019. pp. 415-33.

4. Glass HC, Shellhaas RA, Wusthoff CJ, Chang T, Abend NS, et al; Neonatal Seizure Registry Study Group. Contemporary profile of seizures in neonates: a prospective cohort study. J Pediatr 2016;174:98-103.e1.

5. Symonds JD, Zuberi SM, Stewart K, McLellan A, O’Regan M, et al. Incidence and phenotypes of childhood-onset genetic epilepsies: a prospective population-based national cohort. Brain 2019;142:2303-18.

6. Miceli F, Soldovieri MV, Joshi N, Weckhuysen S, Cooper E, et al. KCNQ2-Related disorders. In: Adam MP, Ardinger HH, Pagon RA, Wallace SE, Bean LJH, et al, editors. GeneReviews ${ }^{\circledR}$ [Internet]. Seattle (WA): University of Washington, Seattle; $1993-2020$.

7. Millichap JJ, Park KL, Tsuchida T, Ben-Zeev B, Carmant L, et al. KCNQ2 encephalopathy: features, mutational hot spots, and ezogabine treatment of 11 patients. Neurol Genet 2016;2:e96.

8. Striano P, Minassian BA. From genetic testing to precision medicine in epilepsy. Neurotherapeutics 2020;17:609-15.

9. Kuersten M, Tacke M, Gerstl L, Hoelz H, Stülpnagel CV, et al. Antiepileptic therapy approaches in KCNQ2 related epilepsy: a systematic review. Eur J Med Genet 2020;63:103628.

10. Biervert C, Schroeder BC, Kubisch C, Berkovic SF, Propping P, et al. A potassium channel mutation in neonatal human epilepsy. Science 1998;279:403-6. 
11. Orhan G, Bock M, Schepers D, Ilina EI, Reichel SN, et al. Dominant-negative effects of KCNQ2 mutations are associated with epileptic encephalopathy. Ann Neurol 2014;75:382-94.

12. Goto A, Ishii A, Shibata M, Ihara Y, Cooper EC, et al. Characteristics of KCNQ2 variants causing either benign neonatal epilepsy or developmental and epileptic encephalopathy. Epilepsia 2019;60:1870-80.

13. Cornet MC, Sands TT, Cilio MR. Neonatal epilepsies: clinical management. Semin Fetal Neonatal Med 2018;23:204-12.

14. Vilan A, Mendes Ribeiro J, Striano P, Weckhuysen S, Weeke LC, et al. A distinctive ictal amplitude-integrated electroencephalography pattern in newborns with neonatal epilepsy associated with KCNQ2 mutations. Neonatology 2017;112:387-93.

15. Scala M, Bianchi A, Bisulli F, Coppola A, Elia M, et al. Advances in genetic testing and optimization of clinical management in children and adults with epilepsy. Expert Rev Neurother 2020;20:251-69.

16. Dilena R, De Liso P, Di Capua M, Consonni D, Capovilla G, et al. Influence of etiology on treatment choices for neonatal seizures: a survey among pediatric neurologists. Brain Dev 2019;41:595-9.

17. Numis AL, Angriman M, Sullivan JE, Lewis AJ, Striano P, et al. KCNQ2 encephalopathy: delineation of the electroclinical phenotype and treatment response. Neurology 2014;82:368-70.

18. Scheffer IE, Berkovic S, Capovilla G, Connolly MB, French J, et al. ILAE classification of the epilepsies: position paper of the ILAE commission for classification and terminology. Epilepsia 2017;58:512-21.

19. Schubert-Bast S, Hofstetter P, Fischer D, Schloesser R, Ramantani G, et al. Sodium channel blockers in KCNQ2-encephalopathy: Lacosamide as a new treatment option. Seizure 2017;51:171-3.

20. Reif PS, Tsai MH, Helbig I, Rosenow F, Klein KM. Precision medicine in genetic epilepsies: break of dawn? Expert Rev Neurother 2017; 17:381-92.

21. Klotz KA, Lemke JR, Korinthenberg R, Jacobs J. Vitamin B6-responsive epilepsy due to a novel KCNQ2 mutation. Neuropediatrics 2017;48:199-204. 